\title{
Teste de Desempenho Escolar: um estudo de levantamento
}

\author{
Luiza Feijó Knijnik - Pontifícia Universidade Católica do Rio Grande do Sul, Porto Alegre/RS, Brasil \\ Claudia Giacomoni - Universidade Federal do Rio Grande do Sul, Porto Alegre/RS, Brasil \\ Lilian Milnitsky Stein - Pontifícia Universidade Católica do Rio Grande do Sul, Porto Alegre/RS, Brasil
}

\begin{abstract}
Resumo
O Teste de Desempenho Escolar (TDE), de 1994, é um instrumento psicométrico de aplicação individual que avalia de forma ampla as capacidades fundamentais para o desempenho escolar em três áreas específicas: leitura, escrita e aritmética, voltado para $1^{\mathrm{a}}$ a $6^{\mathrm{a}}$ séries do Ensino Fundamental. O objetivo do presente estudo foi realizar um levantamento das publicações científicas que utilizaram o TDE de 1994 a 2011. Foi realizada busca nas bases de dados nacionais e internacionais. Os dados de 222 publicações foram analisados, e observou-se um aumento gradativo do número de publicações, em especial no biênio 2008-9 com um total de 73 publicações científicas. Verificou-se que o teste segue apresentando relevância, assim como a importância de atualizar o TDE, tendo em vista que o instrumento é amplamente utilizado em todo o país, porém possui normas desatualizadas e não está adequado à nova realidade de ensino brasileiro (Ensino Fundamental de nove anos).

Palavras-chave: Rendimento escolar; Teste de Desempenho Escolar; Avaliação psicológica.
\end{abstract}

Test for School Achievement: a survey study

\begin{abstract}
The test for School Achievement (TDE), published in 1994, is a psychometric instrument that evaluates basic school achievement skills in three areas: reading, writing and arithmetics, from 1st to 6th grades. The goal of the present study was to make a survey of the scientific publications that used the TDE from 1994 to 2011. National and international databases were included. Data from 222 publications was analyzed, and there was a gradual increase in the number of publications, especially in the 2008-09 biennium, with 73 scientific publications. It was found that the TDE is still very relevant, as well as the importance of updating the TDE, due to its wide usage around the country, but outdated norms and inadequacy to the current reality of Brazilian education (basic education of nine years).

Keywords: Academic Achievement; School achievement Test; Psychological Assessment.
\end{abstract}

\section{Prueba de Rendimiento Académico: un estudio de recopilación}

\begin{abstract}
Resumen
La Prueba de Rendimiento Académico (TDE), 1994, es un instrumento psicométrico para la aplicación individual que evalúa amplias capacidades críticas para el rendimiento escolar en tres áreas específicas: lectura, escritura y aritmética, creado para los estudiantes de primero a sexto grados de la enseñanza primaria. El objetivo de este estudio fue examinar las publicaciones científicas que utilizaron el TDE 1994 hasta 2011. Se realizó una búsqueda en las bases de datos nacionales e internacionales. Los datos de 222 publicaciones se analizaron, y hubo un aumento gradual en el número de publicaciones, sobre todo en los años 2008-9 un total de 73 publicaciones científicas. Se encontró que la prueba sigue mostrando interés, así como la importancia de la actualización de la TDE, ya que el instrumento es ampliamente utilizado en todo el país, pero tiene leyes obsoletas y no se ajusta a la nueva realidad de la educación brasileña (escuela primaria nueve años).

Palabras clave: Logro Educativo; Test de Rendimiento Académico; Evaluación psicológica.
\end{abstract}

A avaliação, no sentido amplo, tem merecido, através dos tempos uma atenção especial em todos os setores da atividade humana. Uma das principais atribuições da avaliação diz respeito a promover a construção de conhecimento, visto que o processo de valoração humana favorece reflexões em busca de aprimoramentos. Na dimensão educacional, a avaliação da construção de conhecimento é ainda mais importante, pois permite que os educadores percebam os avanços e dificuldades que os alunos apresentam, seja de maneira sistemática e planejada, seja de forma assistemática, mediante observações (Ferreira \& Leal, 2007).

Neste sentido, a preocupação acerca da avaliação educacional como ferramenta para o desenvolvimento de metodologias de ensino mais eficazes tem sido refletida no cenário nacional por meio de ações do Ministério da Educação (MEC). Exemplos de algumas dessas ações são a Prova Brasil e o Sistema de
Avaliação do Ensino Básico (SAEB), avaliações em âmbito nacional voltadas ao Ensino Fundamental, que buscam avaliar a qualidade do ensino oferecido pelo sistema educacional brasileiro a partir de testes padronizados (Ministério da Educação, 2008). Outras medidas, com objetivos similares, são o Exame Nacional do Ensino Médio (ENEM) e o Exame Nacional de Cursos (ENADE). Nessa perspectiva, o desenvolvimento e aprimoramento de medidas que possibilitem a verificação do desempenho dos alunos são fatores que podem auxiliar na qualificação tanto de professores, quanto de alunos.

Entretanto, apesar do aumento de interesse na área, a realidade da avaliação de desempenho escolar no Brasil tem sido caracterizada por uma carência de instrumentos cientificamente construídos para nossa realidade de ensino. $\mathrm{O}$ único instrumento psicopedagógico brasileiro voltado para avaliação ampla do desempenho escolar é o Teste de 
Desempenho Escolar (TDE) (Stein, 1994), criado em 1994 (Lúcio, Pinheiro \& Nascimento, 2009), inspirado no Wide Range Achievement Test (Wilkinson, 1993), uma bateria publicada pela Psychological Corporation, cujo objetivo é identificar déficits nas habilidades básicas de leitura, escrita e aritmética (Stein, 1994).

O TDE é um instrumento psicométrico de aplicação individual que avalia de forma ampla as capacidades fundamentais para o desempenho escolar em três áreas específicas: 1) leitura - reconhecimento de palavras isoladas do contexto, 2) escrita - escrita do nome próprio e de palavras contextualizadas, apresentadas sob a forma de ditado e 3) aritmética solução oral de problemas e cálculo de operações aritméticas por escrito. Ele foi concebido e normatizado com o objetivo de avaliar escolares de $1^{\mathrm{a}}$ a $6^{a}$ séries do Ensino Fundamental. Cada um dos subtestes apresenta uma escala de itens em ordem crescente de dificuldade que são apresentados à criança independentemente de sua série. A aplicação do subteste é interrompida pelo aplicador assim que os itens apresentados forem muito difíceis de serem resolvidos (Stein, 1994).

Para a construção de cada um dos subtestes do TDE processos distintos foram desenvolvidos. Estudos de validade de conteúdo foram conduzidos, bem como análises de consistência interna para cada subteste, variando de 0.79 a 0.94 . O coeficiente alfa total foi 0.95 . O processo de normatização foi realizado entre 1993 e 1994 com uma amostra estratificada em três tipos de escolas de Porto Alegre: estaduais, municipais e particulares. Em cada uma das escolas participantes foram testados, em média, 15 sujeitos de cada série ( $\left(\mathrm{da} 1^{\mathrm{a}}\right.$ à $\left.6^{\mathrm{a}}\right)$, escolhidos de forma aleatória dentro do grupo da série a que pertenciam. Uma equipe de sete aplicadores participou da coleta de dados. A aplicação do TDE foi feita de forma individual, observando as condições padrão de iluminação, controle de ruídos e acomodações, que são comuns a outros exames psicopedagógicos. Cada aplicador foi treinado previamente para a aplicação. Os dados normativos do TDE derivam-se das médias dos Escores Brutos (EB) e Desvio Padrão (DP), que foram calculados com base no desempenho das crianças para cada um dos extratos da amostra por série e para a idade cronológica (Stein, 1994).

Desde sua publicação vários anos se passaram e algumas mudanças contextuais ocorreram, tais como a recente mudança no currículo escolar brasileiro, aumentando o período do Ensino Fundamental para nove anos (Ministério da Educação, 2004). Além disso, os padrões para testes psicológicos e educacionais da American Psychological Association, (Adams, 2000; International Test Commission, 2000), indicam que um teste deve ser aperfeiçoado quando surgem novos dados de pesquisa ou ocorrem mudanças significativas na área que tornem o teste inadequado para uso. No mesmo sentido, a resolução 02/2003 do Conselho
Federal de Psicologia - CFP (2003) indica que os dados empíricos das propriedades de um teste psicológico devem ser revisados periodicamente, perdendo a condição de uso e saindo da relação de testes para comercialização caso essa revisão não seja realizada. A revisão de um instrumento é desejável por diversas razões, como por exemplo, a atualização de normas, o aumento da faixa etária de destino, inclusão de outras amostras (clínica, por exemplo), a remoção de itens notadamente antigos e a melhora da efetividade de itens, assim como da validade do teste (Strauss, Spreen $\&$ Hunter, 2000).

Alguns estudos vêm apontando a necessidade de adequar o teste à realidade atual, até mesmo sugerindo modificações, como a pesquisa de Lúcio, Pinheiro e Nascimento (2009). O estudo relatou achados de pesquisa que corroboram a necessidade de reformulação do subteste de leitura do TDE, e possivelmente dos outros subtestes também. As autoras investigaram o impacto da introdução de uma nova classe de erros no subteste de leitura do TDE na distribuição dos escores, avaliando cada participante pelo critério original do manual (EB1), assim como pelo novo critério proposto no estudo (EB2). Foi observado que a adoção do critério EB2 tornou o teste mais discriminativo, indicando que o critério atual é muito permissivo, ainda que a adoção do novo critério não tenha impedido o efeito de teto. As autoras apontam que este efeito seria consequência do excesso de itens fáceis e escassez de palavras difíceis no subteste, os quais seriam capazes de discriminar as habilidades das crianças mais experientes e dos leitores mais capazes. Outro ponto fundamental destacado pelo estudo é a proporção de indivíduos que, sendo considerados normais pelos critérios originais do TDE (obtêm a classificação "média" ou "superior"), recaem na faixa inferior de acordo com o novo critério adotado. Isso significa que existe uma proporção de indivíduos cujas dificuldades podem ter sido camufladas em função do critério de acerto estipulado pelo manual. De maneira similar, os estudos de Garcia e De Rose (2004) e Capellini (2001) verificaram que o TDE mostrou-se insensível para detectar diferenças mais apuradas entre participantes com e sem dificuldades de aprendizagem.

Críticas quanto à regionalização das normas de testes que avaliam o desempenho escolar, como o TDE, foram feitas no estudo de Ferreira e colegas (2012), indicando a necessidade de produção de normas por região para esse instrumento. Este estudo sugeriu também que podem haver diferenças entre os desempenho de alunos da rede pública e privada, diferentemente do que foi encontrado no estudo original (Stein, 1994).

Brito e colegas (2012) apontaram a importância das variáveis idade e escolaridade no desempenho escolar, mediante a utilização do TDE, citando diversos estudos que relacionaram diferentes variáveis 
com o desempenho escolar a partir do uso deste instrumento (Capellini, Tonelotto \& Ciasca, 2004; Cia \& Bahram, 2009; Dias, Enumo \& Turini, 2006; Medeiros, Loureiro, Linhares \& Marturano, 2000; Milani \& Loureiro, 2009; Paiva \& Boruchovitch, 2010; Tonelotto \& cols., 2005). Em suas considerações finais, Brito e colegas (2012) comentam a necessidade de uma revisão tanto do conteúdo quanto das normas do TDE.

Dessa forma, como uma etapa inicial de revisão do TDE, este estudo propõe um levantamento bibliográfico para averiguar a relevância do teste mediante a verificação do volume de pesquisas que o utilizam, assim como averiguar possíveis críticas e sugestões que a literatura possa apontar. Sendo assim, o presente trabalho tem como objetivo apresentar e debater os achados de um levantamento bibliográfico, baseado na literatura científica nacional e internacional no período de 1994 a 2011, acerca do uso do TDE em pesquisas científicas. Buscou-se investigar o volume de publicações científicas que vinham fazendo uso do teste, assim como a qualidade desses estudos. Outras informações, como as regiões brasileiras em que o instrumento vem sendo utilizado, visto que suas normas foram feitas em uma amostra do Rio Grande do Sul, e a forma de uso (se todos os subtestes ou algum em particular), também foram levantadas.

\section{Método}

Foram consultadas as ferramentas de busca de referências científicas nacionais: Google Acadêmico, Scielo, Lilacs, Pepsic e Banco de Teses CAPES. As palavras-chave "Teste de Desempenho Escolar" e "TDE" foram utilizadas. Foi realizada ainda uma busca nas bases de dados internacionais PsycInfo, MetaPress (SpringerLink) e Elsevier (Science Direct), utilizando as mesmas palavras chave. $\mathrm{O}$ período pesquisado foi a partir de 1994 até 2011.

Os critérios para inclusão foram: pertinência do trabalho (isto é, ter utilizado o TDE no estudo, pois muitas ocorrências retornaram apenas com a menção genérica de avaliação de desempenho escolar, sem ter necessariamente utilizado o teste) e presença de referência completa do estudo. Após, as publicações Tabela 1. Número total de ocorrências e número de ocorrências selecionada

\begin{tabular}{lccc}
\hline \multirow{2}{*}{ Base de dados } & \multicolumn{3}{c}{ Palavras-chave } \\
\cline { 2 - 3 } & \multicolumn{2}{c}{ Total de ocorrências por palavra-chave } & \multirow{2}{*}{ Ocorrências selecionadas } \\
\cline { 2 - 3 } & "Teste de Desempenho Escolar" & TDE & 154 \\
\hline Google acadêmico & 474 & 574 & 36 \\
Scielo & 83 & 65 & 23 \\
Lilacs & 41 & 20 & 4 \\
Pepsic & 0 & 5 & 53 \\
Banco de teses Capes & 922 & 91 & 5 \\
Psycinfo & 0 & 14 & 2 \\
Metapress (Springerlink) & 2 & 1117 & 4 \\
Elsevier (Science Direct) & 15 & 103 & \\
\hline
\end{tabular}

selecionadas foram categorizadas nos seguintes tipos: artigos científicos, dissertações de mestrado, teses de doutorado, resumos de anais científicos, trabalhos de conclusão de curso e livro.

As seguintes variáveis de cada publicação foram analisadas: ano de publicação, tipo de publicação, região de realização do estudo, tipo de uso do teste, área da ciência do(s) autor(es), Qualis do periódico no qual o artigo científico foi publicado e número de artigos publicados em periódicos internacionais. Mediante análises estatísticas descritivas as variáveis mencionadas foram analisadas e seus resultados são apresentados a seguir.

\section{Resultados}

Foram verificadas oito ferramentas de busca de referências científicas nacionais e internacionais. A Tabela 1 apresenta o número total de ocorrências geral e o número de ocorrências selecionadas a partir dos critérios de inclusão e exclusão para análise utilizando as palavras-chave "Teste de Desempenho Escolar" e "TDE".

Após a seleção de 222 publicações científicas que preencheram os critérios de inclusão, as referências foram classificadas em: artigos científicos (129), dissertações (49), teses (23), resumos de anais de eventos científicos (18), trabalhos de conclusão de curso (2) e livro (1). Dentre os 129 artigos científicos, foram encontrados nove artigos publicados em periódicos de edição internacional, tais como Brain \& Development e Reading \& Writing (Tabela 2).

A fim de verificar a qualidade dos artigos selecionados, foi analisada a classificação dos periódicos científicos nos quais os estudos foram publicados. Para tanto, foi utilizado o sistema Qualis CAPES Psicologia de 2012. O Qualis CAPES é a análise da qualidade dos periódicos científicos, sendo enquadrados em estratos indicativos da qualidade - A1, o mais elevado; A2; B1; B2; B3; B4; B5; C - com peso zero (CAPES, 2009). Quando o periódico analisado não constava no Qualis da Psicologia, a classificação de outras áreas do conhecimento foi utilizada. $86 \%$ dos periódicos científicos tinham Qualis entre A1 e B2 (100) e 12,9\% tinham Qualis entre B3 e B5 (15). 
Após a verificação da qualidade dos periódicos foi listado o número de artigos por periódico no período do levantamento. O objetivo desta verificação foi investigar se as publicações de Qualis elevado estavam concentradas em apenas algumas revistas específicas, ou se havia uma maior dispersão dos artigos em periódicos de alta qualidade. Observou-se um volume diversificado de periódicos nos estratos A1 a B2, destacando-se: Arquivos de Neuropsiquiatria (Qualis B1), com 11 artigos, Psicologia: Reflexão e Crítica (Qualis A1), com 10 artigos, Psicologia em Estudo (Maringá) (Qualis A2), com 8 artigos, Estudos de Psicologia Campinas (Qualis A2), com 7 artigos, Psicologia: Teoria e Pesquisa (Qualis A1), com 6 artigos, Revista CEFAC - Atualização Científica em Fonoaudiologia e Educação (Qualis B2),
Revista da Sociedade Brasileira de Fonoandiologia (Qualis B1), Paidéia Ribeirão Preto (Qualis A1), com 5 artigos cada, e Estudos de Psicologia Natal (Qualis A2), Interação em Psicologia (Qualis A2) e Pró Fono Revista de Atualização Cientifica (Qualis B2) com 4 artigos.

Buscou-se compreender o panorama de utilização do TDE com a passagem do tempo, a fim de verificar se haveria um volume maior de publicações com o instrumento nos anos mais próximos ao seu lançamento, ou se as publicações com o teste se distribuiriam no tempo de maneira mais uniforme. Dessa forma, o número de publicações científicas elencadas no levantamento no período de 1994 a 2011 foi analisado ano a ano. A Figura 2 apresenta esses dados agrupados por biênio.

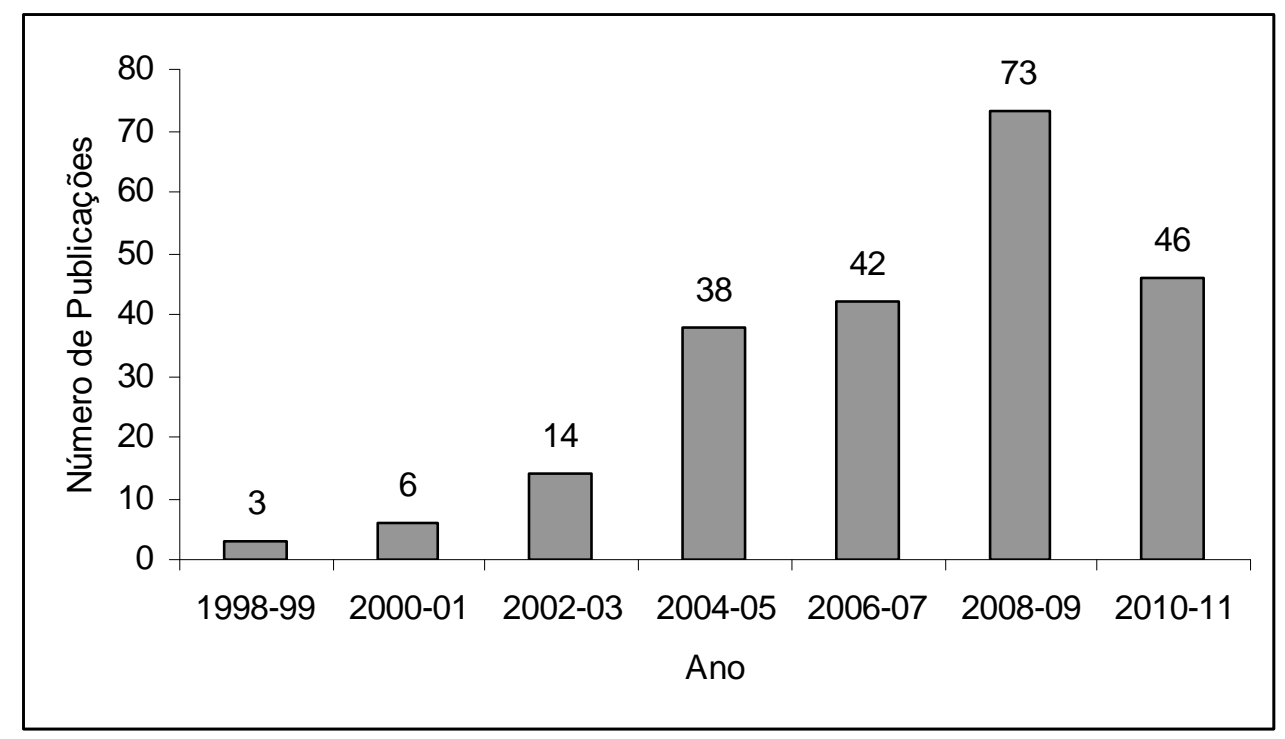

Figura 1. Número de publicações com o TDE por biênio

Ao verificar-se em cada referência bibliográfica $\mathrm{a}(\mathrm{s})$ região(ões) de origem de realização da pesquisa, foi constatado o predomínio das regiões Sul e Sudeste. Destacaram-se os estados de São Paulo, como origem de 63\% das pesquisas, Minas Gerais, 9\% e Rio Grande do Sul, Espírito Santo e Paraná, cada um com 5\% das publicações, seguidos de: Rio de Janeiro (4\%), Santa Catarina (3\%), Distrito Federal (2\%), Mato Grosso $(1 \%)$, Pernambuco (1\%), Roraima (1\%) e Ceará $(1 \%)$.

Tabela 2. Número Total de Ocorrências e Número de Ocorrências Selecionadas

\begin{tabular}{|c|c|c|c|c|c|}
\hline $\begin{array}{l}\text { Periódico } \\
\text { internacional }\end{array}$ & Referência & Ano & $\begin{array}{c}\mathrm{N}^{\mathrm{o}} \text { de } \\
\text { publicações }\end{array}$ & Qualis CAPES & $\begin{array}{l}\text { Fator de } \\
\text { impacto }\end{array}$ \\
\hline $\begin{array}{l}\text { Brain \& } \\
\text { Development }\end{array}$ & $\begin{array}{l}\text { Boscariol e cols. Auditory processing } \\
\text { disorder in patients with language-learning } \\
\text { impairment and correlation with } \\
\text { malformation of cortical development. } \\
67(2-B) \text {, p. } 499-501 \text {. }\end{array}$ & 2011 & 1 & $\begin{array}{c}\text { B1 } \\
\text { (Medicina I) }\end{array}$ & 1.979 \\
\hline \multirow[t]{2}{*}{$\begin{array}{l}\text { Epilepsy \& } \\
\text { Behavior }\end{array}$} & $\begin{array}{l}\text { Fonseca, Tedrus \& Pacheco. Epileptiform } \\
\text { eeg discharges in benign childhood } \\
\text { epilepsy with centrotemporal spikes: } \\
\text { reactivity and transitory cognitive } \\
\text { impairment. 11, p. 65-70. }\end{array}$ & 2007 & \multirow[t]{2}{*}{2} & \multirow[t]{2}{*}{ A2 } & \multirow[t]{2}{*}{1.994} \\
\hline & $\begin{array}{l}\text { Tedrus, Fonseca, Melo \& Ximenes. } \\
\text { Educational problems related to } \\
\text { quantitative eeg changes in benign }\end{array}$ & 2009 & & & \\
\hline
\end{tabular}




\begin{tabular}{|c|c|c|c|c|c|}
\hline & $\begin{array}{l}\text { childhood epilepsy with centrotemporal } \\
\text { spikes. } 15, \text { p. } 486-490 .\end{array}$ & & & & \\
\hline Intelligence & $\begin{array}{l}\text { Colom \& Flores-Mendoza. Intelligence } \\
\text { predicts scholastic achievement irrespective } \\
\text { of SES factors: evidence from Brazil. 35, p. } \\
243-251 .\end{array}$ & 2007 & 1 & A1 & 3.168 \\
\hline $\begin{array}{l}\text { Journal of child and } \\
\text { Adolescent } \\
\text { Psychopharmacology }\end{array}$ & $\begin{array}{l}\text { Farias, Cunha \& Benko. Manganese in } \\
\text { children with attention-deficit hyperactivity } \\
\text { disorder: relationship with methylphenidate } \\
\text { exposure. } 20(2) \text {, p. } 113-118 .\end{array}$ & 2010 & 1 & $\begin{array}{c}\text { B1 } \\
\text { (Medicina I) }\end{array}$ & 2.479 \\
\hline $\begin{array}{l}\text { Journal of Epilepsy } \\
\text { and Clinical } \\
\text { Neurophysiology }\end{array}$ & $\begin{array}{l}\text { Guilhoto e cols. Benign focal seizures of } \\
\text { adolescence and neuropsychological } \\
\text { findings in patients from community. } \\
\text { 15(4), p. 184-191. }\end{array}$ & 2009 & 1 & B4 & não tem \\
\hline $\begin{array}{l}\text { Learning and } \\
\text { Individual } \\
\text { Differences }\end{array}$ & $\begin{array}{l}\text { Rindermann, Flores-Mendoza \& Mansur- } \\
\text { Alves. Reciprocal effects between fluid and } \\
\text { crystallized intelligence and their } \\
\text { dependence on parents' socioeconomic } \\
\text { status and education. } 20 \text {, p. } 544-548 .\end{array}$ & 2010 & 1 & A1 & 1.526 \\
\hline \multirow{2}{*}{$\begin{array}{l}\text { Reading \& } \\
\text { Writing }\end{array}$} & $\begin{array}{l}\text { Correa \& Dockrell. Unconventional word } \\
\text { segmentation in brazilian children's early } \\
\text { text production. } 20 \text {, p. } 815-831 .\end{array}$ & 2007 & \multirow[b]{2}{*}{2} & \multirow[b]{2}{*}{ A2 } & \multirow{2}{*}{1.297} \\
\hline & $\begin{array}{l}\text { Cardoso-Martins \& Silva. Cognitive and } \\
\text { language correlates of hyperlexia: evidence } \\
\text { from children with autism spectrum } \\
\text { disorders. } 23 \text {, p. 129-145. }\end{array}$ & 2010 & & & \\
\hline
\end{tabular}

Com o objetivo de verificar se o TDE vinha sendo utilizado em sua forma completa ou se algum dos subtestes vinha se sobressaindo nos estudos realizados, foi analisada a forma de uso do teste. Assim, verificou-se se o estudo utilizou os três subtestes ou fez uso somente de subtestes específicos: leitura, escrita e/ou aritmética. $83 \%$ das publicações utilizaram o TDE na sua forma completa, e somente três estudos não especificaram a informação de uso.
O TDE não é um instrumento de avaliação de uso exclusivo de psicólogos. Dessa forma, buscou-se verificar quais as áreas da ciência do(s) autor(es) dos trabalhos publicados e selecionados neste levantamento. A Figura 2 apresenta o número de publicações por área da ciência do(s) seu(s) autor(es). Ainda que muitas publicações sejam interdisciplinares, observou-se que $45 \%$ dos estudos são da área da Psicologia.

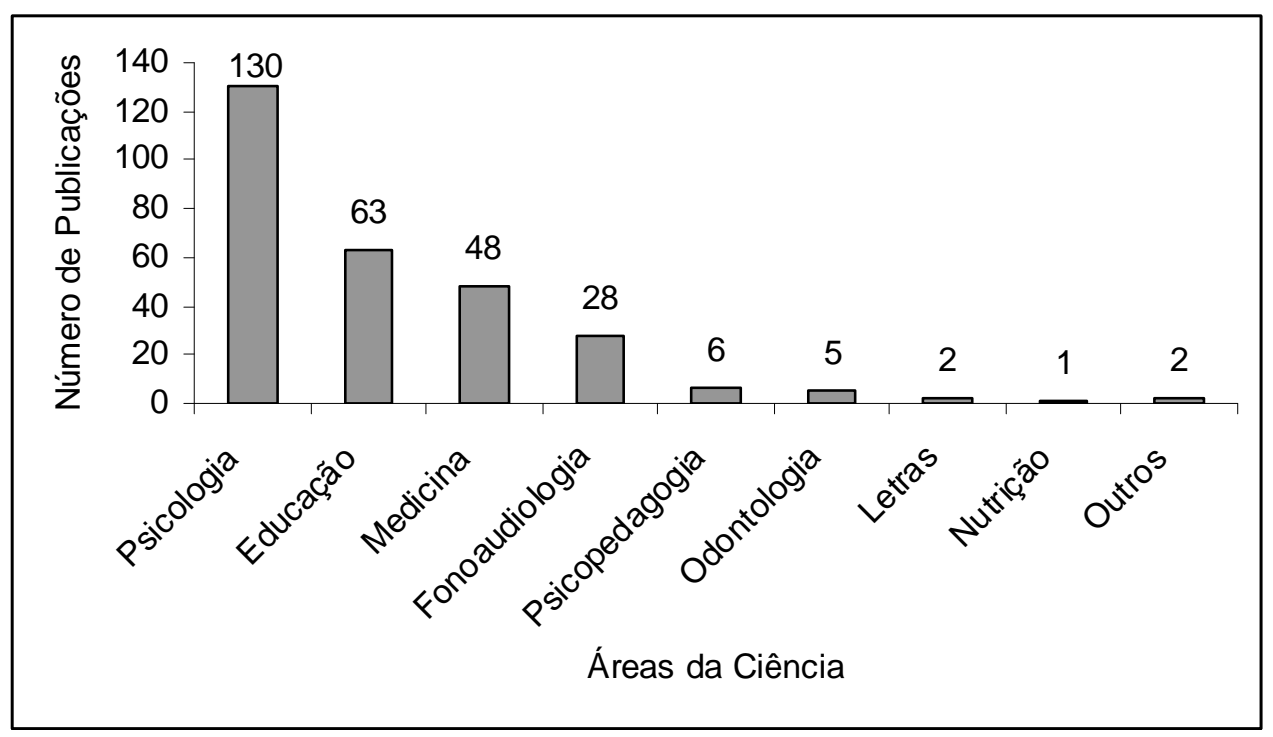

Figura 2. Número de publicações por área da ciência do(s) seu(s) autor(es)

\section{Discussão}

Psico-USF, Bragança Paulista, v. 18, n. 3, p. 407-416, set/dez. 2013
O objetivo deste estudo foi apresentar dados sobre como o TDE vem sendo utilizado a partir de um 
levantamento da literatura científica nacional e internacional no período de 1994 a 2011. Apesar do alto número de retorno de ocorrências com as palavras-chave "Teste de Desempenho Escolar" e "TDE", conforme Tabela 1, nas bases de busca científica verificadas, o trabalho de identificação da literatura cientifica foi criterioso. Destaca-se também a coocorrência da mesma referência em diferentes bases. Cabe salientar que algumas ocorrências com relatos de pesquisas que afirmavam fazer uso do TDE, mas que não estavam disponíveis na íntegra ou não tinham dados completos de referências (por exemplo, um relatório de pesquisa no qual o TDE foi utilizado, mas não constavam os autores) foram eliminadas.

O TDE possui natureza de triagem psicopedagógica, dessa forma, poderia ser feita a suposição de que este seria um instrumento mais voltado para a prática clínica. Entretanto, o levantamento mostrou que do total de 222 referências científicas encontradas, mais de 50\% eram artigos científicos. Esse dado indica que o TDE vem sendo amplamente utilizado como ferramenta em pesquisas interessadas em investigar o desempenho escolar como variável de estudo e/ou controle, conforme demonstrado pelo estudo de Brito e colegas (2012). Observa-se, ainda, o baixo número de resumos de anais de eventos científicos encontrado nas buscas, apontando a restrita disponibilidade desse tipo de material científico online. Esse dado pode ser um indício de que a comunidade acadêmica está tendo pouco acesso ao conteúdo de eventos científicos nacionais.

Ao explorar a qualidade dos periódicos nos quais os artigos científicos levantados foram publicados, verificou-se que $86 \%$ desses periódicos foram classificados entre os Qualis A1 e B2. Este dado aponta indícios da alta qualidade das publicações que vêm fazendo uso do TDE, mesmo que alguns estudos venham apontando a necessidade de revisão desse instrumento (Brito \& cols., 2012; Lúcio, Pinheiro \& Nascimento, 2009).

Observa-se um aumento no volume das publicações que utilizaram o TDE desde sua criação com especial destaque ao biênio 2008-09. Este crescimento parece refletir um crescimento no volume de publicações no Brasil nesse período. Houve registro de um aumento da produção científica brasileira de $56 \%$ de 2007 a 2008, entretanto, uma análise mais profunda revelou que o destaque do biênio 2008-09 pode ter sido influenciado pelo aumento de revistas científicas nacionais indexadas no ISI Web of Science (Meneghini, 2009). Percebe-se que a utilização do TDE em pesquisas seguiu a tendência geral de crescimento, demonstrando que o teste não deixou de ser utilizado com a passagem do tempo, tendo se mantido nas pesquisas científicas.

Em relação à concentração demográfica da origem dos estudos levantados, observou-se a dominância da região Sudeste, mais especificamente, do estado de São
Paulo, e a escassez de estudos nas regiões Norte, Nordeste e Centro-Oeste. Alguns dos fatores que podem contribuir para esses resultados são a extensão continental do Brasil, refletindo em peculiaridades regionais, como a produção intelectual, assim como a localização das universidades que dão origem aos estudos científicos, localizadas na região Sudeste em sua maioria. Este aspecto é considerado relevante por outros levantamentos de literatura similares (Joly, Berberian, Andrade \& Teixeira, 2010; Souza Filho, Belo \& Gouveia, 2006). Destaca-se, no entanto, que a regionalização das normas do TDE não parece impedir que pesquisadores de outros estados o utilizem. Este dado contradiz os achados de Ferreira e colegas (2012), que sugeriram a produção de normas específicas para estados diferentes. Ainda que esta possa ser uma ideia válida, pois permitiria adequar gírias e expressões tipicamente locais a cada região, os achados deste estudo indicam que o TDE tem disso utilizado satisfatoriamente em diferentes regiões brasileiras.

O TDE é composto por três subtestes, quais sejam, leitura, escrita e aritmética, sendo possível realizar aplicação dos subtestes de maneira independente. É razoável supor que com o passar dos anos desde sua criação, algum dos subtestes tenha mostrado mais qualidade do que os demais e, ainda, que algum dos subtestes tenha perdido mais qualidade do que os outros, quanto a discriminação de desempenho, por exemplo. Dessa forma, buscou-se investigar a forma como o teste vem sendo utilizado nos estudos científicos publicados. Verificou-se que a grande maioria das publicações analisadas no levantamento utilizaram o teste completo, indicando que os três subtestes vêm atendendo às demandas da comunidade científica.

Ressalta-se a utilização interdisciplinar do TDE em pesquisa, por áreas afins à psicologia, como a medicina e psicopedagogia, assim como áreas mais distantes, como a odontologia. Apesar de grande parte dos estudos serem da psicologia, é notável que o teste siga tendo o uso que foi proposto na sua criação, de ser um instrumento não exclusivo de psicólogos e que possa auxiliar profissionais de diferentes áreas.

\section{Considerações Finais}

O levantamento realizado neste estudo proporcionou um panorama da utilização do TDE na literatura e apontou indícios da relevância desse instrumento. Verificou-se que mesmo após tantos anos desde a sua publicação e sem sofrer nenhuma atualização, vem sendo amplamente utilizado em pesquisas realizadas em todo o país e em diferentes áreas da ciência. $\mathrm{O}$ crescente volume de publicações também vem indicar que o TDE não ficou obsoleto com o passar dos anos. Entretanto, mesmo com ampla utilização, é interessante refletir sobre a necessidade de revisão desse instrumento. 
A metodologia de levantamento bibliográfico pode ser uma possível limitação deste estudo. A análise da qualidade dos estudos que vêm utilizando o TDE poderia ser mais bem atestada por meio de uma revisão sistemática, técnica mais apropriada para este objetivo específico (Sampaio \& Mancini, 2007).

O presente estudo, de levantamento do uso do TDE desde sua publicação, evidenciou a relevância deste instrumento no cenário de avaliação educacional brasileiro. Os achados quanto ao volume de publicações, áreas da ciência dos pesquisadores e forma de uso (completa ou por subtestes), por exemplo, auxiliaram a caracterizar o status atual do teste. Entretanto, conforme a literatura na área da psicometria indica, a revisão de instrumentos a fim de atualizar normas, realizar estudos de validade e fidedignidade e melhorar itens é altamente desejável (Strauss, Spreen \& Hunter, 2000). Sendo assim, este trabalho alerta para o fato de que o TDE tem sido utilizado com normas não atuais, e que talvez os resultados provenientes deste uso não sejam os mais fidedignos possíveis, conforme apontado por Lúcio, Pinheiro e Nascimento (2009). A discussão realizada no atual levantamento bibliográfico pretende fornecer subsídios para futuros estudos que se proponham a adaptar instrumentos ainda não revisados, em especial o TDE.

\section{Referências}

Adams, K. M. (2000). Practical and ethical issues pertaining to test revisions. Psychological Assessment, 12(3), 281-286.

Brito, L. O., Ambiel, R. A. M., Pacanaro, S. V., Grisard, E., Alves, G. A. S., Rabelo, I. S. \& Leme, I. F. A. S. (2012). Relação das variáveis idade e escolaridade com desempenho escolar de estudantes de ensino fundamental. Avaliação Psicológica, 11(1), 83-93.

Capellini, V. L. M. F. (2001). A inclusão de alunos com necessidades educacionais especiais em classes comuns: avaliação do rendimento acadêmico. Dissertação (Mestrado em Educação Especial) - Centro de Educação e Ciências Humanas, Universidade Federal de São Carlos, São Carlos.

Capellini, S. A., Tonelotto, J. M. F., \& Ciasca, S. M. (2004). Medidas de desempenho escolar: avaliação formal e opinião de professores. Revista Estudos de Psicologia, PUC-Campinas, 21(2), 79-90.

\section{CAPES}

Qualis.

http://www.capes.gov.br/servicos/sala-deimprensa/36-noticias/2550-capes-aprova-a-novaclassificacao-do-qualis. Acessado em 07/abr/2012.

Cia, F. \& Barham, E. J. (2009). Repertório de habilidades sociais, problemas de comportamento, autoconceito e desempenho acadêmico de crianças no início da escolarização. Estudos de Psicologia (Campinas), 26(1), 45-55.

Conselho Federal de Psicologia (2003). Resolução CFP $n^{\circ}$ $002 / 2003$.

Acessado

em

http://www.pol.org.br/pol/export/sites/default/ $\mathrm{pol} /$ legislacao/legislacaoDocumentos/resolucao2 003_02.pdf na data 19/mar/2012.

Dias, T. L., Enumo, S. R. F. \& Turini, F. A. (2006). Avaliação do desempenho acadêmico de alunos do ensino fundamental em Vitória, Espírito Santo. Estudos de Psicologia, 23(4), 381-390.

Ferreira, A. T. B. \& Leal, T. L. (2007). Avaliação na escola e ensino da língua portuguesa: introdução ao tema. Em B. Marcuschi \& L. Suassuna (Org.). Avaliação em língua portuguesa - contribuições para a prática pedagógica. Belo Horizonte: Autêntica.

Ferreira, F. L., Costa, D. S. Micheli, L. R., Oliveira, L. F., Pinheiro-Chagas, P. \& Haase, V G. (2012). School Achievement Test: normative data for a representative sample of elementary school children. Psychology \& Neuroscience, 5(2), 157-164.

Garcia, S. C. \& De Rose, T. M. S. (2004). Rendimento acadêmico e adaptação escolar de alunos participantes na modalidade inclusiva de ensino que combina sala regular e sala de recursos. Revista Brasileira de Educação Especial, Marilia, 10(1), 1-14.

International Test Commission. (2000). Diretrizes para o uso de testes (versão em português publicada pelo Instituto Brasileiro de Avaliação Psicológica, 2003).

Joly, M. C. R. A., Berberian, A. A., Andrade R. G. \& Teixeira, T. C. (2010). Análise de teses e disserações em avaliação psicológica disponíveis na BVS-PSI Brasil. Psicologia: Ciência e Profissão, 30(1), 174-187.

Lúcio, P. S., Pinheiro, A. M. V. \& Nascimento, E. (2009). O impacto da mudança no critério de acerto na distribuição dos escores do subteste de leitura do Teste de Desempenho Escolar. Psicologia em Estudo, Maringá, 14(3), 593-601.

Medeiros, P. C., Loureiro, S. R., Linhares, M. B. M., \& Marturano, E. M. (2000). A auto-eficácia e os aspectos comportamentais de crianças com dificuldade de aprendizagem. Psicologia: Reflexão e Critica, 13(3), 327-336.

Meneghini, R. (2009). Inusitado aumento da produção científica.

http://www.bv.fapesp.br/namidia/noticia/28901 /inusitado-aumento-producao-cientifica/. Acessado em 19/mar/2012.

Milani, R. G. \& Loureiro, S. R. (2009). Crianças em risco psicossocial associado à violência doméstica: 
o desempenho escolar e o autoconceito como condições de proteção. Estudos de Psicologia, 14(3), 191-198.

Ministério da Educação (2004). Ampliação do ensino fundamental para nove anos - relatório do programa. Brasília, DF: Secretaria de Educação Básica.

Ministério da Educação (2008). Plano de Desenvolvimento da Educação - SAEB: ensino médio, matrizes de referência, tópicos e descritores. Brasília: Secretaria de Educação Básica, Instituto Nacional de Estudos e Pesquisas Educacionais Anísio Teixeira.

Paiva, M. L. M. F. \& Boruchovitch, E. (2010). Orientações motivacionais, crenças educacionais e desempenho escolar de estudantes do ensino fundamental. Psicologia em Estudo, Maringá, 15(2), 381-389.

Sampaio, R. F. \& Mancini, M. C (2007). Estudos de revisão sistemática: um guia para síntese criteriosa da evidência científica. Revista Brasileira de Fisioterapia, 11(1), 83-89.

Souza Filho, M. L., Belo, R. \& Gouveia, V. V. (2006). Testes psicológicos: análise da produção científica brasileira no período 2000-2004. Psicologia: Ciência e Profissão, 26(3), 478-489.

Stein, L. M. (1994). TDE - Teste de Desempenho Escolar: manual para aplicação e interpretação. São Paulo, SP: Casa do Psicólogo.

Strauss, E., Spreen, O. \& Hunter, M. (2000). Implications of test revisions for research. Psychological Assessment, 12(3), 237-244.

Tonelotto, J. M. F., Fonseca, L. C., Tedrus, G. M. S. A., Martins, S. M. V., Gibert, M. A. P., Antunes, T. A. \& Pensa, N. A. S. (2005). Avaliação do desempenho escolar e habilidades básicas de leitura em escolares do ensino fundamental. Avaliação Psicológica, 4(1), 33-43.

Wilkinson, G. S. (1993). The Wide Range Achievement Test - third edition. Wilmington, DE: Wide Range.

Recebido em 09/07/2012

Reformulado em 08/05/2013

Aprovado em 12/06/2013

Nota dos autores:

Apoio da Coordenação de Aperfeiçoamento de Pessoal de Ensino Superior.

Sobre as autoras:

Luiza Feijó Knijnik é graduada em Psicologia pela Pontifícia Universidade Católica do Rio Grande do Sul (PUCRS), mestre em Cognição Humana pela mesma instituição e especialista em Neuropsicologia pela Projecto. É doutoranda em Medicina: Ciências Médicas pela Universidade Federal do Rio Grande do Sul (UFRGS). Atua como docente no curso de Psicologia do Cesuca - Faculdade Inedi.

Claudia Giacomoni é graduada em Psicologia pela Pontifícia Universidade Católica do Rio Grande do Sul (PUCRS) (1995), possui mestrado em Psicologia do Desenvolvimento pela Universidade Federal do Rio Grande do Sul (UFRGS) (1997) e doutorado em Psicologia do Desenvolvimento pela UFRGS (2002), com realização de sanduiche na Yale University durante o ano de 1999. É professora adjunta do Departamento de Psicologia do Desenvolvimento e da Personalidade da UFRGS. Possui pesquisas nos seguintes temas: construção de instrumentos de avaliação psicológica, psicologia positiva, bem-estar subjetivo, desenvolvimento infantil, adolescência, satisfação de vida e otimismo. 
Lilian Milnitsky Stein é professora titular do Programa de Pós-Graduação em Psicologia da Pontifícia Universidade Católica do Rio Grande do Sul, com Pós-Doutorado na Universidad de Barcelona, Espanha (2011) e doutorado em Cognitive Psychology - University of Arizona, EUA (1998). É coordenadora do Grupo de Pesquisa em Processos Cognitivos (GPPC, PUCRS), no qual tem realizado pesquisas em Psicologia Experimental Cognitiva, atuando principalmente nos seguintes temas: falsas memórias, emoção e memória e psicologia do testemunho.

Contato com as autoras

Av. Ipiranga, 6681, prédio 11, sala 940 - CEP 90619-900 - Porto Alegre/RS, Brasil.

E-mail: lilian@pucrs.br 
